\title{
Note chorologique sur la flore du Maroc oriental et de ses marges littorales
}

\author{
Mathieu Chambouleyron \\ Emirates Center for Wildlife Propagation, Province de Boulemane, 33250 Missour, Maroc.
}

\section{Correspondencia}

Mathieu Chambouleyron

E-mail: mchambouleyron@yahoo.fr

Recibido: 10 junio 2020

Aceptado: 5 octubre 2020

Publicado on-line: $x x x x 2020$

Editado por: Teresa Navarro

\section{Résumé}

27 taxons sont signalés pour la première fois dans des unités géographiques du Maroc oriental ou de ses marges littorales, six autres y voient leur présence confirmée. De nouvelles données complètent également la répartition de quelques taxons rarement indiqués dans ces unités.

Mots-clés: chorologie, flore vasculaire, Maroc oriental.

\begin{abstract}
Chorological note on the flora of eastern Morocco and its coastline margins
\end{abstract}

27 taxa are reported for the first time in some geographic units of eastern Morocco or its coastal margins, six others are confirmed. New data also complete the known distribution of some taxa rarely indicated in these units.

Keywords: chorology, eastern Morocco, vascular flora.
Des prospections botaniques menées de 2010 à 2019 dans le Maroc oriental et ses marges littorales (Fig. 1) ont permis la découverte de 43 taxons pour la plupart nouveaux localement. Parmi les secteurs prospectés, les monts de Jerada ont la flore la plus méconnue (23 nouveaux taxons observés) et mériteraient un effort de prospection soutenu.

Les échantillons collectés sont conservés dans l'herbier CHAMB. Les codes des unités géographiques sont repris de Fennane \& Ibn Tattou (2005).

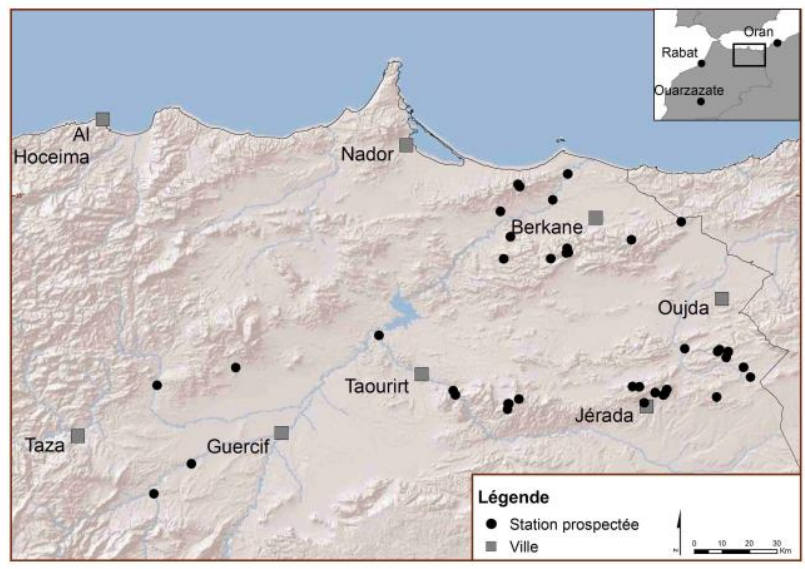

Figure 1. Localités prospectées

Figure 1. Surveyed localities

ANGIOSPERMES DICOTYLEDONES

Amaranthaceae

\section{Amaranthus retroflexus $\mathrm{L}$.}

Op-1: Guercif, Sefsafat (N34.12534, W3.63739), $500 \mathrm{~m}$, culture, 24.X.2019, CHAMB sn.; Taourirt, Melg el ouidane (N34.54423, W3.02484), $210 \mathrm{~m}$, culture, 01.XI.2019, CHAMB sn. Taxon récemment indiqué dans la Maroc oriental, aux environs de Lamrija (Op-1) (Chambouleyron \& al., $2011: 5)$.

\section{Anabasis syriaca Iljin}

Nouveau pour Om-2: Jerada, Jbel Bou Keltoum (N34.34883, W2.09552), $1190 \mathrm{~m}$, matorral, 07.IV.2012, "observation". Taxon largement distribué dans les plaines arides du Maroc oriental, mais restant peu observé sur ses reliefs où il n'est signalé que dans les Bni Snassen (Kahouadji, 1986: 70 ; Fennane \& Ibn Tattou, 2005 : 140).

\section{Asteraceae}

\section{Bidens pilosa $\mathrm{L}$.}

Nouveau pour LM (ici LM-2): Berkane, Aklim (N34.86589, W2.59557), $140 \mathrm{~m}$, culture, 28.IV.2013, CHAMB sn. Taxon exotique, naturalisé et en expansion au Maroc (Ibn Tattou \& Fennane, 2008 : 26).

\section{Erigeron sumatrensis Retz.}

Nouveau pour Op (ici Op-1): Guercif, Tighigha (N34.02684, W3.75982), $590 \mathrm{~m}$, oued, 24.X.2019, CHAMB sn. Taxon exotique, naturalisé au Maroc (Ibn Tattou \& Fennane, $2008: 60$ ). 
Leysera leyseroides (Desf.) Maire

Nouveau pour Om-2: Jerada W2.15785), $1140 \mathrm{~m}$, alfatière,

(N34.32402

07.V.2010, "observation" ; Jerada, Jbel Bou Keltoum (N 34.35600, W2.08957), 1320 m, matorral, 07.IV.2012, "observation". Taxon abondant dans les plaines arides du Maroc oriental, peu cité sur ses reliefs où il est à rechercher selon Fennane (2014a). Quelques données sont fournies dans les environs des Bni Snassen par Blanca (2002) et dans les monts de Debdou par Khattabi (1995: 51, 53) et Chambouleyron \& al. (2015a:6).

\section{Scorzonera hispanica L. subsp. coronopifolia} (Desf.) Rouy

Nouveau pour les monts du Maroc oriental (ici Om2) : Jerada, Tiouli (N34.34327, W1.92130), $1210 \mathrm{~m}$, culture, 19.IV.2014, CHAMB sn. Dans le Maroc oriental, ce taxon n'était indiqué que d'une seule localité vers Oued el himer (Op-1) (Förther \& Podlech, 2001: 91), non loin de là où nous venons de le récolter, alors qu'il est considéré commun par Quézel \& Santa (1963 : 1068) de l'autre côté de la frontière dans le Tell algérien.

\section{Brassicaceae}

\section{Isatis tinctoria L.}

Nouveau pour Om-2: Jerada, Guenfouda, Jbel Mahssor (N34.49775, W1.91169), 1000 m, culture, 01.V.2014, "observation". Relativement bien distribué dans le nord du Maroc oriental, étonnament peu cité localement.

Jonopsidium prolongoi (Boiss.) Batt.

Nouveau pour Om-2: Jerada, Jbel Bou Keltoum (N34.36705, W2.08402), $1600 \mathrm{~m}$, matorral, 07.IV.2012, "observation". Taxon cité non loin d'ici dans son unique localité algérienne au Ghar rouban (Quézel \& Santa, 1962 : 413), ces stations constituant la limite orientale de répartition connue de cette espèce ibéro-maghrébine.

\section{Caryophyllaceae}

Silene apetala Willd.

LM-2 : Zaïo (N34.94895, W2.62842), 130 m, pelouse, 25.III.2018, "observation" ; Berkane, Garma (N34.98631, W2.45640), $40 \mathrm{~m}$, pelouse, 02.IV.2018, "observation". La présence de ce taxon dans LM-2 n'était pas clairement établie, ne disposant que des données de Sennen \& Mauricio (1933: 18) aux environs de Zelouan et Tauima (à la limite LM-1/LM2), et de Talavera (2002: 139, sub S. decipiens Barceló) dans l'aire de Gareb (à la limite LM-1/LM2/Op-1).

Silene colorata subsp. trichocalycina (Fenzl) Maire Om-2: Taourirt, Jbel Bou Khouali (N34.33617, W2.56791), $1720 \mathrm{~m}$, pelouse, 08.V.2011, CHAMB sn. ; Jerada, Guenfouda (N34.50024, W2.02581), $810 \mathrm{~m}$, culture, 05.V.2013, CHAMB sn. La répartition des sous-espèces de $S$. colorata Poir. est à préciser au Maroc, d'après Fennane \& Ibn Tattou (2005: 128).

Spergularia flaccida (Madden) I. M. Turner
Op-1 : Guercif, Mezguitem (N34.43909, W3.49241), 580 m, oued, 23.IV.2018, CHAMB sn. Nous indiquons notre observation du fait de la rareté des données de ce taxon dans $O p$, où seuls Molero \& Montserrat (2006a: 88, sub Spergula fallax (Lowe) E.H.L. Krause) semblent l'avoir récolté, non loin de notre observation entre Guercif et Saka.

\section{Cistaceae}

\section{Helianthemum ruficomum (Viv.) Spreng.}

Nouveau pour Om-2 : Jerada, Touissite (N34.43995, W1.83397), $1010 \mathrm{~m}$, pelouse, 19.IV.2014, CHAMB sn.

\section{Crassulaceae \\ Sedum acre subsp. neglectum (Ten.) Arcang.}

Nouveau pour Om-2: Jerada, Guenfouda, Jbel Mahssor (N34.49123, W1.91853), $1270 \mathrm{~m}$, falaise, 01.V.2014, CHAMB sn. Bien distribué dans les montagnes marocaines, ce taxon n'a été découvert que récemment dans les monts du Maroc oriental, au niveau des monts de Debdou (Chambouleyron \& al., 2015a : 7). II est également indiqué en Algérie dans les monts de Tlemcen (Quézel \& Santa, 1962 : 444), non loin de notre récolte.

\section{Euphorbiaceae}

Chrozophora tinctoria (L.) A. Juss.

Présence potentielle dans le Maroc oriental (ici Op1) : Guercif, Msoun (N34.38131, W3.74890), 600 m, bord de route, 24.X.2019, CHAMB sn. II serait souhaitable de confirmer la présence de ce taxon dans Op-1 par d'autres récoltes plus à l'Est, cette localité étant proche du Rif oriental où ce taxon est déjà connu.

\section{Euphorbia serrata L.}

Nouveau pour Om-2 : Jerada, Touissite (N34.49172, W1.88367), $1010 \mathrm{~m}$, culture, 19.IV.2014, "observation" ; Jerada, Guenfouda, Jbel Mahssor (N34.49775, W1.91169), 1000 m, culture, 01.V.2014, "observation". Peu de données récentes de ce taxon au Maroc d'après Fennane (2018 : 42).

\section{Fabaceae}

\section{Ononis laxiflora Desf.}

Nouveau pour Om-2: Jerada, Guenfouda, Jbel Mahssor (N34.49329, W1.91752), 1120 m, matorral, 01.V.2014, "observation".

\section{Ononis reclinata subsp. mollis (Savi) Bég.}

Nouveau pour Om-2: Jerada (N34.32402, W2.15785), $1140 \mathrm{~m}$, alfatière, 07.V.2010, "observation" ; Jerada, Guenfouda (N34.50024, W2.02581), $810 \mathrm{~m}$, culture, 05.V.2013, "observation" ; Jerada, Jbel Bou Keltoum (N34.35714, W2.12218), $1250 \mathrm{~m}$, matorral, 20.IV.2014, "observation".

\section{Geraniaceae}

Erodium microphyllum Pomel

Nouveau pour Om-2: Jerada, Jbel Bou Keltoum (N34.34883, W2.09552), $1190 \mathrm{~m}$, matorral, 07.IV.2012, CHAMB sn. ; Jerada, Jbel Bou Keltoum 
(N 34.35600, W2.08957), $1320 \mathrm{~m}, \quad$ matorral, 07.IV.2012, CHAMB sn.

\section{Erodium cf tordylioides (Desf.) L'Hér.}

Nouveau pour Om-2: Taourirt (N34.34962, W2.77508), $620 \mathrm{~m}$, rocher, 01.V.2013, CHAMB sn. Taxon signalé de l'autre côté de la frontière dans les monts de Tlemcen (Algérie) par Quézel \& Santa (1963: 576).

Sur l'individu que nous avons prélevé, les méricarpes présentent un sillon infrafovéolaire (glanduleux) bien net, caractère non indiqué par Guittoneau (1972: 112-115) et Ibn Tattou (2007) pour qui ce taxon en est dépourvu. Cela dit, selon Navarro (2015 : 345) la taille et le nombre de sillons seraient variable, allant de zéro à deux. Guittoneau (2010 : 38) indique quant à lui la présence possible d'une ébauche de pli chez ce taxon.

E. mouretii Pit., taxon morphologiquement très proche, présentant un sillon infrafovéolaire, aurait une écologie différente en étant inféodé aux roches quartzitiques (Guittoneau, 1972 : 116 ; 2010 : 38 ; Ibn Tattou, 2007 : 270 ; Navarro, 2015 : 347).

Si $E$. mouretii est strictement inféodé à ces roches, alors ce taxon ne peut pas être celui que nous avons récolté sur les falaises calcaires de Taourirt. L'étude génétique du taxon de cette localité serait nécessaire pour valider notre détermination (G.G. Guittonneau, com. pers.), $E$. mouretii et $E$. tordylioides étant génétiquement individualisés (Fiz-Palacios \& al., 2010).

Erodium trifolium (Cav.) Guitt.

Nouveau pour Om-2: Taourirt, Jbel Bou Khouali (N34.32095, W2.59994), 1430 m, rocher, 08.V.2011, CHAMB sn. ; Taourirt, Jbel Bou Khouali (N34.33617, W2.56791), $1720 \mathrm{~m}$, rocher, 08.V.2011, "observation" ; Jerada (N34.37722, W2.19658), $1480 \mathrm{~m}$, rocher, 20.IV.2014, "observation" ; Jerada, Guenfouda, Jbel Mahssor (N34.49123, W1.91853), $1270 \mathrm{~m}$, falaise, 01.V.2014, "observation".

\section{Orobanchaceae}

Orobanche gracilis $\mathrm{Sm}$.

Nouveau pour Om-2 : Jerada, Touissite (N34.48938, W1.88414), $1110 \mathrm{~m}$, matorral, 19.IV.2014, CHAMB sn.

\section{Papaveraceae}

\section{Papaver argemone L. subsp. argemone}

Nouveau pour le Maroc oriental (ici Om-2) : Jerada, Guenfouda, Jbel Mahssor (N34.49123, W1.91853), $1270 \mathrm{~m}$, pelouse, 01.V.2014, CHAMB sn.

\section{Platycapnos tenuiloba Pomel subsp. tenuiloba}

Nouveau pour Om-2 : Jerada, Jbel Bou Keltoum (N 34.35600, W2.08957), 1320 m, matorral, 07.IV.2012, CHAMB sn.

\section{Sarcocapnos crassifolia (Desf.) DC. subsp. crassifolia}

Om-2 : Jerada (N34.37722, W2.19658), $1480 \mathrm{~m}$, rocher, 20.IV.2014, CHAMB sn. ; Jerada, Guenfouda, Jbel Mahssor (N34.49123, W1.91853), $1270 \mathrm{~m}$, falaise, 01.V.2014, "observation". Dans le Maroc oriental, ce taxon a été indiqué par Jahandiez \& Maire (1932 : 260), puis Maire (1965:17), au Ghar rouban, un massif à cheval entre l'Algérie et le Maroc, mais dont l'essentiel est situé côté algérien dans le prolongement de Om-2. II est également signalé dans Om-2 par Fennane \& Ibn Tattou (2005: 308) sans indication de localité.

S. crassifolia subsp. simplicifolia Chamboul., Bidat \& J.F. Léger est un autre taxon, récemment décrit des monts de Debdou (Chambouleyron \& al., 2015b), qui se caractérise par ses feuilles simples ( $v s$ composées pour la subsp. crassifolia). Son isolement géographique est très relatif et surtout lié aux faibles capacités de dissémination de S. crassifolia. Sur ce point, il est intéressant de noter que nos nouvelles données de subsp. crassifolia sont situées à seulement $70 \mathrm{~km}$ d'une population de subsp. simplicifolia des monts de Debdou. Au regard de la proximité géographique des deux taxons, la particularité morphologique de la subsp. simplicifolia est intriguante.

\section{Plantaginaceae}

\section{Chaenorhinum flexuosum (Desf.) Lange}

Nouveau pour Om-2: Taourirt (N34.34962, W2.77508), $620 \mathrm{~m}$, rocher, 01.V.2013, "observation". A récolter pour détermination infraspécifique, Dobignard (2009: 70) distinguant deux sous-espèces (flexuosum et maroccanum (Pau) Dobignard).

Linaria tristis (L.) Mill.

Om-2: Taourirt, Jbel Bou Khouali (N34.32095, W2.59994), $1430 \mathrm{~m}$, rocher, 08.V.2011, CHAMB sn. ; Jerada, Guenfouda, Jbel Mahssor (N34.49123, W1.91853), $1270 \mathrm{~m}$, falaise, 01.V.2014, CHAMB sn.

Coloration des corolles variable, allant du blanc au jaunâtre, veinées de violet, éperon conforme ou plus franchement jaune, protubérances tâchées de violet ou blanc-jaunâtre. La détermination infraspécifique est problématique car nos récoltes présentent des corolles et éperons relativement longs (respectivement $26-30 \mathrm{~mm}$ et $14-17 \mathrm{~mm}$ long), valeurs intermédiaires entre les subsp. marginata (Desf.) Maire et mesatlantica D. A. Sutton, d'après les valeurs indiquées par Sutton (1988: 377). D'après Dobignard (2009: 73), la subsp. marginata est absente du Maroc.

Plantago major subsp. intermedia (Gilib.) Lange Op-1: Taourirt (N 34.36379, W2.78222), $440 \mathrm{~m}$, oued, 01.V.2013, CHAMB sn. Dans le Maroc oriental, ce taxon n'a été signalé que par Molero \& Montserrat (2006b : 171) dans les environs de Guercif (Op-1).

Détermination basée uniquement sur les caractères séminaux, avec sur notre échantillon des capsules à 22-26 graines, ces dernières mesurant c. $1 \mathrm{~mm}$ long (pas tout à faire mûres).

\section{Plumbaginaceae}

Limonium echioides (L.) Mill.

Nouveau pour Om-1: Berkane, Tafoughalt, Dar moussa (N34.79382, W2.61779), $510 \mathrm{~m}$, matorral, 28.IV.2013, "observation". A noter que la donnée de 
Molero \& Montserrat (2006b : 164) dans l'aire des "Beni Snassen" se rapporte à LM-2.

\section{Polygonaceae}

Polygonum argyrocoleum Steud. ex Kuntze

Op-1: Guercif (N34.32784, W3.28507), 340 m, culture, 01.XI.2019, CHAMB sn. Au Maroc ce taxon n'est connu que de quelques récoltes de la région de Guercif-Taourirt (Pastor, 1996: 230 ; Molero \& Montserrat, 2006c : 23).

\section{Ranunculaceae}

\section{Ranunculus fibrosus Pomel}

Om-2: Jerada, Tiouli (N34.40745, W1.81124), $1120 \mathrm{~m}$, alfatière, 19.IV.2014, CHAMB sn. (dét. A. Dobignard) ; Jerada (N34.37616, W2.17332), $1300 \mathrm{~m}$, pelouse, 20.IV.2014, CHAMB sn. La chorologie marocaine des trois taxons du groupe $R$. paludosus (R. paludosus Poir., $R$. flabellatus Desf., $R$. fibrosus Pomel) reste à clarifier (Dobignard, 2009 : 10), raison pour laquelle nous indiquons nos données. Ajoutons tout de même que le lectotype de R. fibrosus Pomel provient du Ghar rouban (Dobignard, 2009: 8), massif frontalier algéromarocain très proche de notre première récolte.

\section{Ranunculus paludosus Poir.}

Om-1 : Berkane, Tafoughalt (N34.81533, W2.40579), $750 \mathrm{~m}$, matorral, 27.IV.2013, CHAMB sn. (dét. A. Dobignard). Malgré le signalement de $R$. paludosus dans Om par Fennane \& Ibn Tattou (2005 : 332), nous $\mathrm{y}$ indiquons notre récolte, ces auteurs n'ayant pas retenu $R$. flabellatus et $R$. fibrosus.

Nos spécimens présentent des racines napiformes non ou peu revêtues de restes fibreux.

\section{Rubiaceae}

Galium verticillatum Danthoine

Nouveau pour Om-2: Taourirt, Jbel Bou Khouali (N34.32059, W2.60310), $1390 \mathrm{~m}$, rocher, 08.V.2011, "observation".

\section{Valerianaceae}

\section{Centranthus battandieri Maire}

Nouveau pour Om-2: Jerada (N34.37722, W2.19658), $\quad 1480 \mathrm{~m}, \quad$ rocher, 20.IV.2014, "observation". Taxon connu dans le massif du Ghar rouban en Algérie (Quézel \& Santa, 1963 : 883), de l'autre côté de la frontière à proximité de notre observation.

\section{ANGIOSPERMES MONOCOTYLEDONES \\ Asparagaceae}

Ornithogalum narbonense $\mathrm{L}$.

Nouveau pour Om-2: Taourirt, Jbel Bou Khouali (N34.30355, W2.60470), $1360 \mathrm{~m}$, culture, 08.V.2011, CHAMB sn.

\section{Prospero obtusifolium (Poir.) Speta}

Om-1 : Berkane, Tafoughalt (N34.81383, W2.41279), $820 \mathrm{~m}$, matorral, 25.XI.2012, CHAMB sn. La présence de ce taxon dans les monts du Maroc oriental méritait confirmation d'après Fennane (2014b : 387), bien qu'il ait été signalé dans les environs des Beni-Snassen par Jahandiez \& Maire (1931 : 130) et Mateos \& Valdés (2002: 860).

\section{Cyperaceae}

\section{Carex divisa Huds.}

Nouveau pour Om-2: Jerada, Guenfouda, Jbel Mahssor (N34.49123, W1.91853), $1270 \mathrm{~m}$, pelouse, 01.V.2014, "observation".

\section{Iridaceae}

\section{Romulea ramiflora Ten.}

Nouveau pour Om-2: Taourirt, Jbel Bou Khouali (N34.30355, W2.60470), 1360 m, culture, 18.III.2012, CHAMB sn.

\section{Liliaceae}

Tulipa sylvestris subsp. australis (Link) Pamp.

Nouveau pour LM-2: Berkane, Ras el ma (N35.07077, W2.40834), 80 m, matorral, 02.IV.2018, "observation" ; Zaïo (Kebdana) (N35.03004, W2.56502), $760 \mathrm{~m}$, matorral, 02.IV.2018,

"observation".

\section{Orchidaceae}

Anacamptis coriophora (L.) R. M. Bateman, Pridgeon \& M. W. Chase

Om-1: Berkane, Tafoughalt, Dar moussa (N34.79382, W2.61779), $510 \mathrm{~m}$, matorral, 28.IV.2013, CHAMB sn. La présence de ce taxon dans les monts du Maroc oriental méritait confirmation d'après El Oualidi \& Fennane (2014a : 443).

\section{Ophrys fusca Link}

Om-1 : Berkane, Tafoughalt (N34.79425, W2.46388), $830 \mathrm{~m}$, matorral, 25.III.2018, CHAMB ; Berkane, Tafoughalt (N34.82891, W2.41035), 660 m, matorral, 02.IV.2018, "observation". La présence de ce taxon dans les monts du Maroc oriental méritait confirmation d'après El Oualidi \& Fennane (2014b : 447).

\section{Ophrys tenthredinifera Willd.}

Om-1: Berkane, Aïn Regadal (N34.85608, W2.20005), $1190 \mathrm{~m}$, yeuseraie, 09.V.2010, "observation" ; Berkane, Tafoughalt (N34.79425, W2.46388), $830 \mathrm{~m}$, matorral, 25.III.2018, CHAMB sn.; Oujda, Ahfir, Col de Garbouz (N34.91519, W2.03688), $550 \mathrm{~m}$, pinède, "observation"

Om-2 : Jerada, Touissite (N34.47203, W1.89025), 1010 m, matorral, 19.IV.2014, CHAMB sn.

La présence de ce taxon dans les monts du Maroc oriental méritait confirmation d'après El Oualidi \& Fennane (2014b : 449). De l'autre côté de la frontière, Quézel \& Santa (1962: 228) l'indiquent commun dans le Tell algérien, tout comme Babali \& al (2018 : 54-56) dans les monts de Tlemcen.

\section{Serapias lingua $\mathrm{L}$.}

LM-2 : Zaïo (Kebdana) (N35.03727, W2.57044), $740 \mathrm{~m}$, matorral, 02.IV.2018, "observation". La présence de ce taxon dans LM méritait confirmation d'après El Oualidi \& Fennane (2014c : 446).

\section{Poaceae}

Poa ligulata Boiss. 
Nouveau pour Om-2: Taourirt, Jbel Bou Khouali (N34.33617, W2.56791), $1720 \mathrm{~m}$, rocher, 08.V.2011, CHAMB sn.

\section{Remerciements}

Nous adressons nos vifs remerciements à MM. Alain Dobignard qui a identifié nos récoltes de Ranunculus fibrosus et $R$. paludosus, et a très aimablement accepté de relire le manuscrit de cet article, GuyGeorges Guittonneau pour les remarques à propos des Erodium tordylioides et $E$. mouretii, et Errol Véla pour avoir confirmé nos identifications d'Ophrys fusca. Nous remercions également l'Emirates Center for Wildlife Propagation (ECWP) et l'International Fund for Houbara Conservation (IFHC) pour la mise à disposition de leurs ressources (matériel, fonds bibliographique et herbier).

\section{Références}

Babali, B., Medjahdi, B. \& Bouazza, M. (2018). Les orchidacées de la région de Tlemcen (Algérie). Acta Botanica Malacitana, 43, 43-62.

Blanca, G. (2002). Leysera L. in Valdés, B et al. (eds.), Catalogue des plantes vasculaires du Nord du Maroc, incluant des clés d'identification, 2, 649. CSIC, Madrid.

Chambouleyron, M., Bidat, M., Ibn Tattou, M., Molero, J., Montserrat, J.-M., Pyke, S. \& Léger, J.-F. (2015a). Contribution à la connaissance de la flore vasculaire du Maroc oriental : plaine de Lamrija et revers nord des monts de Debdou. Bulletin de I'Institut Scientifique de Rabat, Section Sciences de la Vie, 37, 1-16.

Chambouleyron, M., Bidat, M. \& Léger, J.-F. (2015b). Sarcocapnos crassifolia subsp. simplicifolia (Papaveraceae, Fumarioideae), a new narrowendemic taxon from northeastern Morocco. Annales Botanici Fennici, 52, 205-210.

Dobignard, A. (2009). Contributions à la connaissance de la flore du Maroc et de l'Afrique du Nord. Nouvelle série 2. La flore du Nord-Maroc. Journal de botanique, 46-47, 1-136.

El Oualidi, J. \& Fennane, M. (2014a). Orchis L. In M. Fennane et al. (eds), Flore Pratique du Maroc, 3, 440-443. Travaux de I'Institut Scientifique, Série Botanique, 40, Rabat.

El Oualidi, J. \& Fennane, M. (2014b). Ophrys L. In M. Fennane et al. (eds), Flore Pratique du Maroc, 3, 446-451. Travaux de l'Institut Scientifique, Série Botanique, 40, Rabat.

El Oualidi, J. \& Fennane, M. (2014c). Serapias L. In M. Fennane et al. (eds), Flore Pratique du Maroc, 3, 444-446. Travaux de l'Institut Scientifique, Série Botanique, 40, Rabat.

Fiz-Palacios, O., Vargas, P., Vila, R., Papadopulos, A.S.T. \& Aldasoro, J.A. (2010). The uneven phylogeny and biogeography of Erodium (Geraniaceae): radiations in the Mediterranean and recent recurrent intercontinental colonization.
Annals of Botany, 106(6), 871-884.

Guittoneau, G.-G. (1972). Contribution à l'étude biosystématique du genre Erodium L'Hér. dans le Bassin Méditerranéen occidental. Boissiera, 20, 7155.

Guittoneau, G.-G. (2010). Aperçu sur la taxonomie, l'écologie et la phylogénie du genre Erodium L'Hér. dans le bassin méditerranéen. Edité par l'auteur.

Fennane, M. (2014a). Leysera L. In M. Fennane et al. (eds), Flore Pratique du Maroc, 3, 198. Travaux de I'Institut Scientifique, Série Botanique, 40, Rabat.

Fennane, M. (2014b). Prospero Salisb. In M. Fennane et al. (eds), Flore Pratique du Maroc, 3, 386-387. Travaux de l'Institut Scientifique, Série Botanique, 40, Rabat.

Fennane, M. (2018). Éléments pour un Livre rouge de la flore vasculaire du Maroc, 5. Dicotyledonae (Cactaceae - Euphorbiaceae) (Version 1). Editions Tela-Botanica, Montpellier.

Fennane, M. \& Ibn Tattou, M. (2005). Flore vasculaire du Maroc : inventaire et chorologie, Pteridophyta, Gymnospermae, Angiospermae p.p., 1. Travaux de I'Institut Scientifique, Série Botanique, 37, Rabat.

Förther, H. \& Podlech, D. (2001). Contributions to the Flora of Northern Africa, I. New and noteworthy taxa. Sendtnera 7, 85-91.

Ibn Tattou, M. (2007). Erodium L'Hér. ex Aiton. In M. Fennane et al. (eds), Flore Pratique du Maroc, 2, 267-275. Travaux de l'Institut Scientifique, Série Botanique, 38, Rabat.

Ibn Tattou, M. \& Fennane, M. (2008). Flore vasculaire du Maroc : inventaire et chorologie, Asteraceae et Monocotylédones, 2. Travaux de l'Institut Scientifique, Série Botanique, 39, Rabat.

Jahandiez, E. \& Maire, R. (1931). Catalogue des plantes $d u$ Maroc (Spermatophytes et Ptéridophytes), Ptéridophytes, Gymnospermes et Monocotylédones, 1. Minerva, Alger.

Jahandiez, E. \& Maire, R. (1932). Catalogue des plantes du Maroc (Spermatophytes et Ptéridophytes), Dicotylédones Archichlamydées, 2. Minerva, Alger.

Kahouadji, A. (1986). Recherches floristiques sur le massif montagneux des Beni-Snassene (Maroc oriental). Thèse Université des Sciences et Techniques du Languedoc, Montpellier.

Khattabi, A. (1995). Étude phytoécologique et phytosociologique des matorrals du massif des Béni Snassen, de la chaîne des Horsts et des monts de Debdou (Maroc Oriental). Thèse Faculté des Sciences, Oujda.

Maire, R., (1965). Flore de l'Afrique du Nord, Dicotyledonae: Rhoeadales: Papaveraceae, sf. Fumarioidea p.p.; Capparidaceae, Cruciferae p.p., 12. Le Chevalier, Paris.

Mateos, M.A. \& Valdés, B. (2002). Scilla L. in Valdés, $\mathrm{B}$ et al. (eds.), Catalogue des plantes vasculaires du Nord du Maroc, incluant des clés d'identification, 2, 859-860. CSIC, Madrid. 
Molero, J. \& Montserrat, J.-M. (2006a). Novedades corologicas y taxonomicas selectas para el catalogo de la flora vascular del norte de Marruecos. Lagascalia, 26, 83-110.

Molero, J. \& Montserrat, J.-M. (2006b). Aportaciones a la flora vascular de las comarcas orientales del Norte de Marruecos. Lagascalia, 26, 155-177.

Molero, J. \& Montserrat, J.-M. (2006c). Quenopodiáceas nuevas o raras para la flora de Marruecos. Lagascalia, 26, 7-24.

Navarro, C. (2015). Erodium L'Hér. in Castroviejo S. (coord.), Flora Iberica, RhamnaceaePolygalaceae, 9, 316-372. Real Jardín Botánico, C.S.I.C., Madrid.

Pastor, J. (1996). Nuevas citas para la flora de
Marruecos. Lagascalia, 18, 230-231.

Quézel, P. \& Santa, S. (1962-1963). Nouvelle flore de l'Algérie et des régions désertiques méridionales, 2 vols. CNRS, Paris.

Sennen, E.L. \& Mauricio, H. (1933). Catálogo de la flora del Rif Oriental y principalmente de las cabilas limítrofes con Melilla. Gráficas La Ibérica, Melilla.

Sutton, D.A., (1988). A revision of the tribe Antirrhineae. British Museum (Natural History) \& Oxford university Press, London.

Talavera, S., (2002). Silene L. in Valdés, B. et al. (eds.), Catalogue des plantes vasculaires du Nord du Maroc, incluant des clés d'identification, 2, 132144. CSIC, Madrid. 